Virginia Commonwealth University VCU Scholars Compass

2004

\title{
The Surgical Infection Society Guidelines on Antimicrobial Therapy for Intra-Abdominal Infections: An Executive Summary
}

John E. Mazuski

Saint Louis University

Robert G. Sawyer

University of Virginia

Avery B. Nathens

University of Washington

See next page for additional authors

Follow this and additional works at: http://scholarscompass.vcu.edu/pharmacy_dean_pubs

Part of the Pharmacy and Pharmaceutical Sciences Commons

This is a copy of an article published in Surgical Infections (C) 2004 copyright Mary Ann Liebert, Inc.; Surgical Infections is available online at: http://online.liebertpub.com.

\section{Downloaded from}

http://scholarscompass.vcu.edu/pharmacy_dean_pubs/11

This Article is brought to you for free and open access by the Office of the Dean at VCU Scholars Compass. It has been accepted for inclusion in Publications from the Office of the Dean by an authorized administrator of VCU Scholars Compass. For more information, please contact libcompass@vcu.edu. 
Authors

John E. Mazuski, Robert G. Sawyer, Avery B. Nathens, Joseph T. DiPiro, Moshe Schein, Kenneth A. Kudsk, and Charles Yowler 


\title{
The Surgical Infection Society Guidelines on Antimicrobial Therapy for Intra-Abdominal Infections: An Executive Summary
}

\author{
JOHN E. MAZUSKI, ${ }^{1}$ ROBERT G. SAWYER, ${ }^{2}$ AVERY B. NATHENS, ${ }^{3}$ JOSEPH T. DIPIRO, ${ }^{4}$ \\ MOSHE SCHEIN, ${ }^{5}$ KENNETH A. KUDSK, ${ }^{6}$ and CHARLES YOWLER ${ }^{7}$ FOR THE \\ THERAPEUTIC AGENTS COMMITTEE OF THE SURGICAL INFECTION SOCIETY
}

\begin{abstract}
The Surgical Infection Society last published guidelines on antimicrobial therapy for intraabdominal infections in 1992 (Bohnen JMA, et al., Arch Surg 1992;127:83-89). Since then, an appreciable body of literature has been published on this subject. Therefore, the Therapeutics Agents Committee of the Society undertook an effort to update the previous guidelines, primarily using data published over the past decade. An additional goal of the Committee was to characterize its recommendations according to contemporary principles of evidencebased medicine. To develop these guidelines, the Committee carried out a systematic search for all English language articles published between 1990 and 2000 related to antimicrobial therapy for intra-abdominal infections. This literature was reviewed individually and collectively by the Committee, and categorized according to the type of study and its quality. Additional articles published prior to 1990 were also utilized when necessary. By a process of iterative consensus, the Committee developed provisional guidelines for antimicrobial therapy for intra-abdominal infections based on this evidence. Following extensive review by members of the Society, these guidelines were approved for publication in final form by the Council of the Surgical Infection Society. This executive summary delineates the Society's current recommendations for antimicrobial therapy of patients with intra-abdominal infections. Topics discussed include the selection of patients needing therapeutic antimicrobials, duration of antimicrobial therapy, acceptable antimicrobial regimens, and identification and treatment of higher-risk patients. Guidelines for patient selection and specific antimicrobial regimens were based on relatively good evidence, but those regarding optimal duration of therapy and treatment of higher-risk patients relied mostly on expert opinion, since there was a paucity of high-quality studies on those issues. Relevant areas for future investigation include the safety, convenience, and cost-effectiveness of available antimicrobial regimens for lower-risk patients, and better means for identifying and treating higher-risk patients with intra-abdominal infections.
\end{abstract}

From the Departments of Surgery, Saint Louis University School of Medicine, St. Louis, MO, ${ }^{1}$ University of Virginia, Charlottesville, VA, ${ }^{2}$ University of Washington, Seattle, WA, ${ }^{3}$ Bronx Lebanon Hospital Center, Bronx, NY, ${ }^{5}$ University of Tennessee, Memphis, $\mathrm{TN}^{6}{ }^{6}$ Case Western Reserve University, Cleveland, $\mathrm{OH}^{7}{ }^{7}$ and the College of Pharmacy, University of Georgia, and Department of Surgery, Medical College of Georgia, Augusta, GA. ${ }^{4}$

Present affiliation for Doctor Mazuski: Department of Surgery, Washington University School of Medicine, St. Louis, MO. Present affiliation for Doctor Kudsk: Department of Surgery, University of Wisconsin, Madison, WI.

The article was prepared by the Therapeutic Agents Committee of the Surgical Infection Society, and approved by the Council of the Surgical Infection Society as an official position paper of the organization. 


\section{INTRODUCTION}

A NTIMICROBIAL THERAPY for intra-abdominal infections is an important adjunct to surgical or radiologically guided procedures designed to gain control of the infected focus. In 1992, the Antimicrobial Agents Committee of the Surgical Infection Society published a set of guidelines on the use of antimicrobials for the treatment of intra-abdominal infections [1]. Since these initial guidelines were published, a number of additional studies have provided further insight into the use of antimicrobials for these infections. In 2000, the Therapeutic Agents Committee of the Surgical Infection Society, the successor to the Antimicrobial Agents Committee, was given the task of updating the original guidelines.

As part of this revision, the Committee not only reviewed and revised the previous guidelines, but also formally categorized its recommendations using the principles of evidencebased medicine. The published studies used to create these guidelines were categorized as Class I, in which evidence was obtained from a prospective randomized controlled trial or a meta-analyses of such trials; Class II, in which evidence came from other prospective and retrospective studies containing clearly reliable data; and Class III, in which evidence was from uncontrolled studies, case reports, and expert opinion (Table 1). Guidelines were then assigned a level based upon the strength of the supporting evidence. Level 1 guidelines are those supported by high-quality research-based evidence, generally prospective randomized controlled trials. Level 2 guidelines are those supported by less rigorously designed trials and retrospective controlled studies, and Level 3 guidelines are those supported primarily by uncontrolled trials and expert opinion (Table 2) [2,3].
Several mechanisms were used to ensure that these guidelines reflected the consensus of the membership of the Surgical Infection Society. The preliminary guidelines proposed by the Committee were presented at the Annual Meeting of the Surgical Infection Society held May $3-5,2001$. Two independent reviewers from the Society provided in-depth critiques of the proposed guidelines, and an open forum allowed other members of the Society to comment on them as well. Based on these critiques and discussion, the final guidelines were formulated and presented for review to the Council of the Surgical Infection Society, which approved their publication.

This document represents the executive summary of the final guidelines approved by the Council. A more extensive discussion of the evidence behind these recommendations is provided in an additional document [4]. In addition, the reader is referred to the original guidelines developed by Bohnen et al. [1] for additional information, since the current guidelines were designed to update, but not supplant the previous recommendations.

The guidelines presented here focus on several specific issues related to the use of antimicrobials for patients with intra-abdominal infections, each of which will be discussed separately:

1. Which patients require therapeutic administration of antimicrobials because of intra-abdominal infections? How do we distinguish patients with contamination, requiring only prophylactic antibiotics, from those with established intra-abdominal infections?

2. How long should antimicrobial agents be administered to patients with intra-abdominal infections?

3. What antimicrobial regimens are recommended for the treatment of patients with

Table 1. Class of Evidence

\begin{tabular}{ll}
\hline Class & Evidence \\
\hline I & $\begin{array}{l}\text { Prospective randomized controlled trials or meta-analyses of such trials. } \\
\text { II }\end{array}$ \\
$\begin{array}{l}\text { Prospective studies without randomization or other studies in which data were collected prospectively, } \\
\text { and retrospective analyses based on clearly reliable data. These include observational studies, cohort } \\
\text { studies, prevalence studies, and retrospective case control studies. }\end{array}$ \\
III & Uncontrolled studies using retrospective data, such as clinical series or case reviews, and expert opinion.
\end{tabular}

Adapted from [2] and [3]. 
Table 2. Rating Scale for Recommendations

\begin{tabular}{ll}
\hline Level & Recommendation \\
\hline 1 & Recommendation based on good research-based evidence. Supported primarily by Class I data, although \\
& strong Class II data may be the basis of the recommendation when the issue is not amenable to study \\
with a prospective randomized controlled trial. & Recommendation based on fair research-based evidence. Supported by limited data from prospective, \\
& randomized controlled trials, or from other prospective or retrospective analyses with good study \\
design, and strongly supported by expert opinion. & \\
& Recommendation based primarily on limited or uncontrolled data, and supported by expert opinion. \\
\hline
\end{tabular}

Adapted from [2] and [3].

intra-abdominal infections? Are any regimens of greater or lesser efficacy?

4. What risk factors can be used to identify patients likely to fail initial antimicrobial therapy? Should the antimicrobial regimen be intensified in such patients to decrease the risk of failure?

\section{PATIENT SELECTION}

The scope of intra-abdominal infections covered by the previous and present guidelines are those generally described as secondary or tertiary peritonitis and intra-abdominal abscess. Infections that are not considered in these guidelines include primary peritonitis and infections associated with indwelling intra-abdominal catheters, primary genitourinary or gynecological disorders, and localized infections of an abdominal organ for which no operative or other procedure is performed.

These guidelines apply to the use of therapeutic antimicrobials for established intra-abdominal infections, and not to the use of prophylactic antimicrobials to prevent surgical site infections following abdominal procedures. Although the distinction between therapeutic and prophylactic use of antimicrobials is usually apparent, there are patients in whom antimicrobial use falls into a gray area. Such patients include those who have significant intra-abdominal contamination sustained shortly before or during an operative procedure, and those who have an infected site within the abdominal cavity confined to a specific organ, which can be excised surgically. A number of studies have attempted to determine which of these patients can be treated with what are es- sentially prophylactic antimicrobials, given for $24 \mathrm{~h}$ or less, and which patients require longerterm therapeutic antimicrobial therapy.

The best evidence that antimicrobials given for $24 \mathrm{~h}$ or less are adequate for many patients with intra-abdominal contamination comes from Class I studies of patients with traumatic bowel perforations, in whom surgical therapy was undertaken without undue delay [5-7]. A similar principle should apply to iatrogenic bowel perforations operated on immediately, such as endoscopic perforations of the colon or enterotomies occurring during surgical interventions. There is also limited evidence that patients with gastroduodenal perforations operated on early do not require therapeutic antimicrobials [8]. However, the consensus opinion is that patients with small bowel or colonic perforations greater than $12 \mathrm{~h}$ old, or gastroduodenal perforations greater than $24 \mathrm{~h}$ old, have established intra-abdominal infections and should be treated with therapeutic antimicrobials.

Patients with an inflamed or infected focus that can be eradicated at the time of surgical intervention are another group that can potentially be treated with short-term prophylactic antimicrobials. Class II evidence indicates that antimicrobial therapy for $24 \mathrm{~h}$ or less is adequate for patients with acute or gangrenous appendicitis, acute or gangrenous cholecystitis, and those with bowel necrosis due to a vascular accident or strangulating bowel obstruction, in whom there is no evidence of perforation or infected peritoneal fluid [8,9]. However, this does not apply to patients whose infection has extended beyond the initial anatomic focus or who have intra-operative findings of purulent, infected peritoneal fluid. Since these patients 
have established peritonitis, therapeutic antimicrobial therapy is warranted. Table 3 lists the types of problems for which therapeutic antimicrobials are not considered necessary.

\section{Summary of recommendations}

1. Patients with peritoneal contamination due to traumatic or iatrogenic bowel injuries repaired within $12 \mathrm{~h}$ (Level 1) and those having gastroduodenal perforations less than $24 \mathrm{~h}$ old (Level 3) are not considered to have established intra-abdominal infections, and should be treated with prophylactic antimicrobials for $24 \mathrm{~h}$ or less.

2. Patients with a fully removable focus of inflammation, such as those with acute or gangrenous, but non-perforated appendicitis or cholecystitis, and those with bowel necrosis or obstruction without perforation or peritonitis, should be treated with prophylactic antimicrobials for $24 \mathrm{~h}$ or less (Level 2).

3. Patients with more extensive conditions than those noted above should be treated as having established infections, and given therapeutic antimicrobials for greater than $24 \mathrm{~h}$ (Level 3).

\section{DURATION OF ANTIMICROBIAL THERAPY}

The optimal duration of antimicrobial therapy for intra-abdominal infections remains controversial, in part because there are no large Class I trials that have evaluated this issue specifically. Recommendations are therefore based on limited data and expert opinion.

There is growing consensus that shorter, rather than prolonged, courses of antimicrobial therapy are appropriate for most patients with

Table 3. Conditions for which Therapeutic Antimicrobials $(>24 \mathrm{H})$ Are Not Recommended

\footnotetext{
Traumatic and iatrogenic enteric perforations operated on within $12 \mathrm{~h}$

Gastroduodenal perforations operated on within $24 \mathrm{~h}$ Acute or gangrenous appendicitis without perforation Acute or gangrenous cholecystitis without perforation

Transmural bowel necrosis from embolic, thrombotic, or obstructive vascular occlusion without perforation or established peritonitis or abscess
}

intra-abdominal infections. These shorter antimicrobial courses decrease the exposure of the patient to costly and potentially toxic agents and may diminish the spread of resistant organisms within the hospital. The previous guidelines recommended 5-7 days of antimicrobial therapy for most patients with intra-abdominal infections [1], and some authorities have proposed even shorter durations of therapy [10].

Two general approaches that limit the duration of antimicrobial therapy have been advocated. One approach relies on protocols that specify the length of antimicrobial therapy based on intra-operative findings at the time of the initial surgical intervention. Patients with limited intraperitoneal infections receive only 2 days of antimicrobial therapy, whereas those with more extensive peritonitis receive up to 5 days of therapy. Two Class II studies found no increase in failure rates relative to historical data when antimicrobials were limited to a maximum of 5 days using such protocols [8,9].

The alternative approach uses the patient's symptoms and signs to guide the duration of antimicrobial therapy. This approach is based on observational studies that demonstrated a low risk of treatment failure in patients who were afebrile and had normal white blood cell counts at the time of cessation of antimicrobial therapy $[11,12]$. In addition, one Class I and one Class II study demonstrated that discontinuation of antimicrobials at the time of resolution of clinical signs was as successful as a fixed duration of antimicrobial therapy, and resulted in a shorter duration of antibiotic use [13,14].

It is fairly common to observe prolonged courses of antimicrobial therapy being used in patients with persistent fevers or elevated white blood cell counts. Observational studies revealed that such patients were at high risk for treatment failure. However, those studies also showed that persistent clinical signs usually indicated an ongoing source of infection, which would optimally have been treated with further surgical intervention rather than prolongation of antimicrobial therapy $[11,12]$. There are no prospective trials demonstrating that longer courses of antimicrobials improve outcome in most patients with persistent symptoms after initial management of their intra-abdominal in- 
fections. Thus, the consensus opinion is that ongoing clinical evidence of infection should trigger a careful search for a focus of infection in the abdomen or elsewhere, rather than prolongation of antimicrobial therapy.

There are very limited Class III data, however, that a longer course of antimicrobial treatment is warranted in selected patients in whom optimal source control cannot be achieved. In a retrospective analysis of patients treated with open abdominal techniques for persistent bacterial peritonitis, a shorter duration of antimicrobial therapy was associated with higher morbidity [15]. Longer courses of therapy may also be reasonable in patients with extensive necrotizing infections of the retroperitoneum, in whom primary source control is not feasible, or in patients with tertiary peritonitis, who have repeatedly failed attempts at treatment.

\section{Summary of recommendations}

1. Antimicrobial therapy of most established intra-abdominal infections should be limited to no more than 5 (Level 2) to 7 days (Level 3). The duration of antimicrobial therapy for intra-abdominal infections can be based on the intra-operative findings at the time of initial intervention (Level 3). Antimicrobial therapy can be discontinued in patients when they have no clinical evidence of infection such as fever or leukocytosis (Level 2).

2. Continued clinical evidence of infection at the end of the time period designated for antimicrobial therapy should prompt appropriate diagnostic investigations rather than prolongation of antimicrobial treatment (Level 3).

3. If adequate source control cannot be achieved, a longer duration of antimicrobial therapy may be warranted (Level 3).

\section{RECOMMENDED ANTIMICROBIAL REGIMENS}

The fundamental principle of antimicrobial therapy for intra-abdominal infections is to utilize agents effective against aerobic/facultative
Table 4. Recommended Antimicrobial Regimens for PATIENTS With InTRA-AbDOMinAl InfECtions

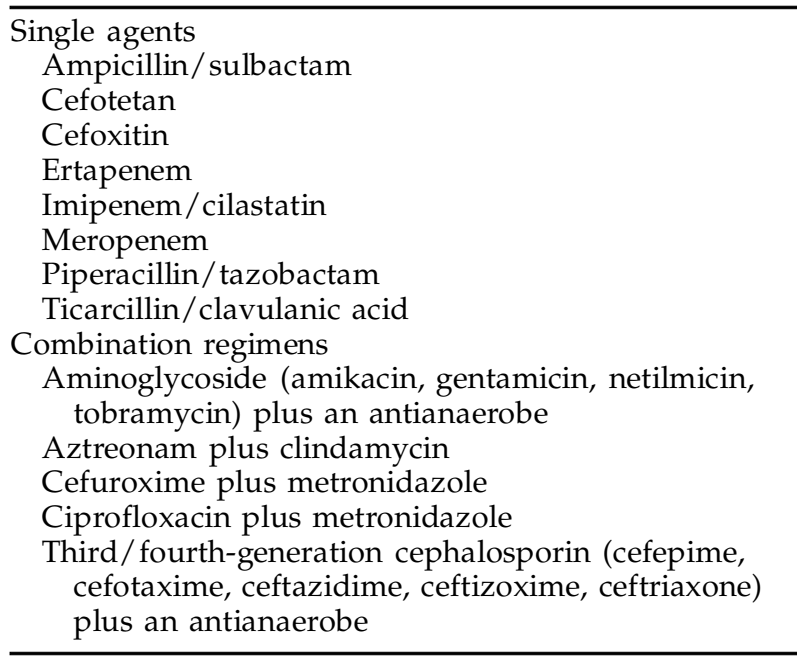

anaerobic Enterobacteriacae and anaerobic organisms, particularly Bacteroides fragilis. Bohnen et al. [1] identified a number of single agents or combination regimens that were effective for the treatment of patients with these infections. Based on a large body of Class I evidence [16-72], additional agents can be added to that list. Recommended single agents now include secondgeneration cephalosporins with anaerobic coverage, beta-lactam/beta-lactamase inhibitor agents, and carbapenems (imipenem/cilastatin, meropenem, and ertapenem). Recommended combination regimens include cefuroxime or a third- or fourth-generation cephalosporin plus an antianaerobic agent (either clindamycin or metronidazole), aztreonam plus clindamycin, ciprofloxacin plus metronidazole, or an aminoglycoside plus an antianaerobe (Table 4). It is likely that other agents will be added to this list in the future as prospective trials of newer agents are completed and published.

The published literature provides little guidance in selecting a specific regimen. Antimicrobial trials have generally been designed to demonstrate therapeutic equivalence, and have not been powered adequately to demonstrate superiority. Further, most patients entered into those trials have had community-acquired infections such as perforated appendicitis and have not been severely ill. Since all antimicrobial regimens appear to be of approximately equal efficacy for less severely ill patients with 
community-acquired infections, agents that are less toxic, less expensive, and have a narrower spectrum of activity would be preferable for these patients. Examples of such agents include second-generation cephalosporins with anaerobic coverage, ampicillin/sulbactam, and ticarcillin/clavulanic acid.

The use of oral antibiotics to complete the antimicrobial course seems reasonable based on Class I and Class III evidence. Conversion from intravenous to oral ciprofloxacin plus metronidazole was found equivalent to a mandatory intravenous regimen in one prospective trial [56]. These oral agents, as well as oral amoxicillin/clavulanic acid, have been utilized in other trials as well $[34,64,69]$. In these studies, oral antibiotics were used to follow initial intravenous therapy when patients were able to tolerate an oral diet.

In recent years, fewer patients with intraabdominal infections are being treated with aminoglycoside-based regimens, in part due to concerns of toxicity. However, of equal concern is aminoglycoside underdosing and failure to attain therapeutic serum concentrations, potentially resulting in treatment failure [27]. Serum aminoglycoside concentrations should therefore be monitored carefully when these antibiotics are utilized in divided daily doses. Once-daily administration of aminoglycosides may avoid problems of underdosing. In a large meta-analysis of patients with any type of gram-negative infection, this regimen was shown to be of equal efficacy compared to divided daily dose regimens, and possibly associated with fewer side effects [73]. Two prospective trials also found that once daily administration of aminoglycosides was efficacious in patients with intra-abdominal infections [74,75].

The need for treatment of Enterococcus sp. remains controversial. Prospective trials comparing carbapenems or beta-lactam/beta-lactamase inhibitor combinations against regimens that do not provide enterococcal coverage have not demonstrated improved clinical outcome with the former regimens $[17,18,26,27,30,32,34$, $36,38,41,42,48,51-57,61,62,67,69,70,72]$. Thus, for most patients with community-acquired infections, enterococcal coverage should not be used as a criterion for selection of the antimicrobial regimen.
The usefulness of intra-operative cultures to guide therapy of patients with intra-abdominal infections, particularly those with communityacquired infections, is highly questionable. There are no prospective studies demonstrating that alteration of an initial empiric antimicrobial regimen on the basis of culture results decreases the incidence of treatment failure. Although changing to different antimicrobial agents is sometimes attempted in patients having ongoing signs of clinical infection, such patients would more likely be benefited by an expeditious search for the source of the persistent infection than by altering the initial antimicrobial regimen.

\section{Summary of recommendations}

1. Antimicrobial regimens for intra-abdominal infections should cover common aerobic and anaerobic enteric flora. The following antimicrobials or combinations of antimicrobials are effective for the treatment of intra-abdominal infections. No regimen has been demonstrated to be superior to another (Level 1).

Single agents:

Ampicillin/sulbactam

Cefotetan

Cefoxitin

Ertapenem

Imipenem/cilastatin

Meropenem

Piperacillin/tazobactam

Ticarcillin/clavulanic acid

Combination regimens:

Aminoglycoside (amikacin, gentamicin, netilmicin, tobramycin) plus an antianaerobe Aztreonam plus clindamycin

Cefuroxime plus metronidazole

Ciprofloxacin plus metronidazole

Third/fourth-generation cephalosporin (cefepime, cefotaxime, ceftazidime, ceftizoxime, ceftriaxone) plus an antianaerobe (clindamycin or metronidazole)

2. For less severely ill patients with community-acquired infections, antimicrobial agents having a narrower spectrum of activity, such as antianaerobic cephalosporins, ampicillin/sulbactam, or ticarcillin/clavulanic acid, are preferable to more costly 
agents having broader coverage of gramnegative organisms and/or greater risk of toxicity (Level 3).

3. Completion of an antimicrobial course with oral forms of ciprofloxacin plus metronidazole (Level 2) or with oral amoxicillin/ clavulanic acid (Level 3) is acceptable in patients able to tolerate an oral diet.

4. Once-daily administration of aminoglycosides is the preferred dosing regimen for patients receiving these agents for intraabdominal infections (Level 2). Careful attention should be paid to prompt attainment of therapeutic antibiotic concentrations if divided daily doses of aminoglycosides are used (Level 3).

5. Regimens providing enterococcal coverage are not necessary for the treatment of most patients with community-acquired intra-abdominal infections (Level 2).

6. The routine use of intra-operative cultures is controversial. There is no evidence that altering the antimicrobial regimen on the basis of intra-operative culture results improves outcome (Level 3).

\section{ANTIMICROBIAL THERAPY FOR THE HIGHER-RISK PATIENT}

The risks of treatment failure and death as a result of intra-abdominal infections are highly variable. A younger patient with a localized perforation of the appendix has a much lower risk than an elderly individual with diffuse peritonitis secondary to a colonic perforation [76]. However, the precise identification and optimal treatment of the higher-risk patient remains problematic.

Multivariate analyses have identified a number of risk factors for treatment failure and death in patients with intra-abdominal infections. Most of these factors relate to the patient's underlying physiological status and response to the infection. Thus, advanced age, poor nutritional status, a low serum albumin concentration, preexisting medical disorders such as significant cardiovascular disease, and, in particular, a higher APACHE II score are significantly associated with treatment failure and death [77-83]. However, some studies identi- fied risk factors that related more to the treatments rendered than to the patient's underlying condition. For instance, the failure to achieve adequate source control with the initial operative procedure increased the risk of an adverse clinical outcome [84]. Of particular relevance to the selection of antimicrobial agents were risk factors related to the actual organisms identified in the peritoneal cavity. Several studies suggested that infections involving resistant organisms, particularly those likely to be acquired in the hospital, increased the risks of treatment failure and death $[55,57,84,85]$.

It is difficult to provide definitive recommendations with regard to antimicrobial therapy of higher-risk patients. Efforts to develop consensus guidelines are hampered by the fact that most Class I evidence comes from antimicrobial trials in lower-risk patients with community-acquired infections, with resultant mortality and failure rates well below those observed in epidemiological surveys of patients with intra-abdominal infections. Thus, most recommendations regarding antimicrobial therapy for higher risk patients are, of necessity, based on expert opinion.

As in the previous guidelines [1], it is recommended that higher-risk patients be treated with antimicrobial regimens having a broader spectrum of activity against most gram-negative aerobic/facultative anaerobic organisms. Such regimens include extended-range beta-lactam/ beta-lactamase agents such as piperacillin/ tazobactam, carbapenems such as imipenem/ cilastatin or meropenem, third- or fourth-generation cephalosporins plus an antianaerobic agent, aztreonam plus clindamycin, and ciprofloxacin plus metronidazole. The use of an aminoglycoside plus an antianaerobe is another alternative, although an adequate dosing regimen must be employed (Table 5). Even though resistant gram-negative organisms such as Pseudomonas sp. are more commonly encountered in higher-risk patients, several prospective trials found that the routine addition of an aminoglycoside to another agent effective against these organisms conferred no additional benefit [86-88].

Failure due to Enterococcus appears to be much more common in higher-risk patients $[84,89]$. In contrast to the recommendation re- 
Table 5. Recommended Antimicrobial Regimens for Higher-Risk Patients with Intra-Abdominal Infections

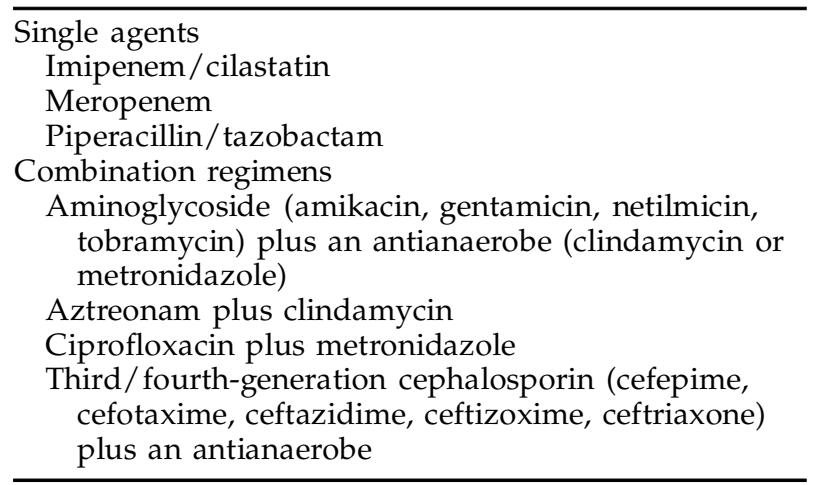

garding lower-risk patients with communityacquired infections, it seems reasonable to recommend the use of agents having enterococcal coverage for higher-risk patients likely to have enterococcal infections. Nonetheless, this recommendation must be considered tentative, since definitive studies in higher-risk patients have not been performed.

The development of fungal peritonitis is also more common in higher-risk patients [90,91]. In one prospective randomized trial, prophylactic therapy with fluconazole improved outcome in patients at high risk for Candida peritonitis [92]. However, there is some controversy regarding the adequacy of fluconazole for the treatment of established candidal infections in the peritoneal cavity, and some authorities recommend amphotericin B as the preferred agent instead of fluconazole for these patients [93]. Ultimately, the choice of antifungal therapy will be heavily influenced by the risks of toxicity in a given patient.

Patients with tertiary peritonitis, who have ongoing intra-abdominal infection after previous attempts at control, are quite difficult to treat. The organisms that these patients harbor include coagulase-negative Staphylococcus, enterococci (sometimes resistant to vancomycin), multiply resistant gram-negative bacilli, and fungal organisms [94,95]. Empiric antimicrobial therapy should be directed at the nosocomial pathogens likely to be present, based on the patient's history of previous antimicrobial therapy and the institutional history of likely nosocomial pathogens and their resistance patterns. Empiric antimicrobials should be adjusted according to definitive culture results.
Ultimately, many of these patients will succumb to or with their infections, regardless of the adequacy of antimicrobial therapy [94-96].

\section{Summary of recommendations}

1. In patients with intra-abdominal infections, treatment failure and death is associated with patient-related risk factors such as advanced age, poor nutritional status, a low serum albumin concentration, and preexisting medical conditions, especially significant cardiovascular disease. A higher APACHE II score is the most consistently recognized risk factor for both death and treatment failure (Level 1).

2. Disease- and treatment-related risk factors, including a nosocomial origin of infection, the presence of resistant pathogens, and the lack of adequate source control are associated with treatment failure and death (Level 2).

3. Patients at higher risk for failure (particularly from non-community-acquired organisms) should be treated with an antimicrobial regimen having a broader spectrum of coverage of gram-negative aerobic/facultative anaerobic organisms (Level 3):

Single agents:

Imipenem/cilastatin

Meropenem

Piperacillin/tazobactam

Combination regimens:

Aminoglycoside (amikacin, gentamicin, netilmicin, tobramycin) plus an antianaerobe (clindamycin or metronidazole)

Aztreonam plus clindamycin

Ciprofloxacin plus metronidazole

Third/fourth-generation cephalosporin (cefepine, cefotaxime, ceftazidime, ceftizoxime, ceftriaxone) plus an antianaerobe

4. Routine addition of an aminoglycoside to other agents having broad spectrum gramnegative coverage, such as imipenem/cilastatin, piperacillin/tazobactam, or third/ fourth generation cephalosporins, provides no additional benefit (level 2).

5. Higher-risk patients likely to fail due to Enterococcus, such as those of advanced age, higher APACHE II scores, a non-appendiceal source of infection, a postoperative infection, or a nosocomial origin of infection 
may benefit from the use of a regimen covering this organism (Level 3).

6. Addition of empiric antifungal therapy with fluconazole is reasonable for patients with postoperative intra-abdominal infections at high risk for candidiasis (Level 2). For patients with established Candida peritonitis, antifungal therapy with amphotericin B may be preferable to the use of fluconazole, but the choice of therapy must be influenced by the risk of toxicity in a given patient (Level 3).

7. Patients with tertiary peritonitis are likely to harbor difficult to eradicate organisms, such as coagulase negative staphylococci, enterococci (including vancomycin-resistant enterococci), multiply resistant gram-negative bacilli, and yeast (Level 2). Empiric therapy should be directed at organisms likely to be present based on the patient's history of previous antimicrobial therapy and local patterns of infectious organisms and resistance, and modified according to definitive culture results (Level 3).

\section{RECOMMENDATIONS FOR FUTURE RESEARCH}

The development of these guidelines has led to the identification of several issues that could be addressed successfully by future investigators. These issues can be separated into those that involve lower-risk patients with community-acquired infections, and those that involve more severely ill patients, particularly those with postoperative or hospital-acquired infections.

With regard to patients with community-acquired intra-abdominal infections, there are already a number of suitable antimicrobial regimens available, all of which appear to be of approximately equivalent efficacy. Given the reasonably good success rates of the current regimens, it seems unlikely that future study will uncover a regimen of substantially greater efficacy. Patients with community-acquired infections would more likely be benefited by identifying which regimens are the most convenient, cost-effective, and least toxic without compromising safety. To that end, one key area for further investigation is to determine defin- itively how long antimicrobial therapy needs to be given to lower-risk patients with community-acquired infections. Further delineation of the role for oral antimicrobial regimens, which can be easily administered outside of the hospital setting, could also further the goal of finding more cost-effective regimens for the treatment of these patients.

With regard to the higher-risk patient, improvement in efficacy should remain the major focus of future investigations. These patients, particularly those with hospital-acquired infections, still have poor clinical outcomes and many succumb to the infectious process or its sequelae. Development of criteria by which these higher-risk patients could be identified readily would facilitate future investigations. However, of greater importance would be an increased inclusion of higher-risk patients in antimicrobial trials, from which many are presently excluded. Ideally, clinical studies involving only higher-risk patients would be performed. If this were not feasible, adequate numbers of higher-risk patients should be enrolled and stratified in standard trials, with the results of these higher-risk patients being analyzed separately. The development of newer antimicrobial agents for the treatment of these patients would also be important, given the rapid colonization of hospitalized patients with resistant pathogens. Improvement of antimicrobial regimens for higher-risk patients would not only provide benefits to the patients themselves, but might diminish the outbreak of multiply-drug-resistant microorganisms, the selection of which is highly encouraged through the use of inappropriate or ineffective antimicrobials.

\section{REFERENCES}

1. Bohnen JMA, Solomkin JS, Dellinger EP, et al. Guidelines for clinical care: anti-infective agents for intraabdominal infection. A Surgical Infection Society policy statement. Arch Surg 1992;127:83-89.

2. Pasquale, MD. Practice management guidelines for trauma [On-line]. Trauma Practice Guidelines. Available: www.east.org, 1998.

3. Wolfe BM, Mathiesen KA. Clinical practice guidelines in nutrition support: can they be based on randomized clinical trials? JPEN 1997;21:1-6.

4. Mazuski JE, Sawyer RG, Nathens AB, et al. The Sur- 
gical Infection Society guidelines on antimicrobial therapy for intra-abdominal infections: evidence for the recommendations. Surg Infect 2002; 3:175-233.

5. Fabian TC, Croce MA, Payne LW, et al. Duration of antibiotic therapy for penetrating abdominal trauma: a prospective trial. Surgery 1992;112:788-795.

6. Bozorgzadeh A, Pizzi WF, Barie PS, et al. The duration of antibiotic administration in penetrating abdominal trauma. Am J Surg 1999;177:125-131.

7. Kirton OC, O'Neill PA, Kestner M, et al. Perioperative antibiotic use in high-risk penetrating hollow viscus injury: a prospective randomized, double-blind, placebo-control trial of 24 hours versus 5 days. J Trauma 2000;49:822-832.

8. Schein M, Assalia A, Bachus H. Minimal antibiotic therapy after emergency abdominal surgery: a prospective study. Br J Surg 1994;81:989-991.

9. Andåker L, Höjer H, Kihlström E, et al. Stratified duration of prophylactic antimicrobial treatment in emergency abdominal surgery. Metronidazole-fosfomycin vs. metronidazole-gentamicin in 381 patients. Acta Chir Scand 1987;153:185-192.

10. Wittmann DH, Schein M. Let us shorten antibiotic prophylaxis and therapy in surgery. Am J Surg 1996;172:26S-32S.

11. Lennard ES, Minshew BH, Dellinger EP, et al. Leukocytosis at termination of antibiotic therapy: its importance for intra-abdominal sepsis. Arch Surg 1980;115:918-921.

12. Lennard ES, Dellinger EP, Wertz MJ, et al. Implications of leukocytosis and fever at conclusion of antibiotic therapy for intra-abdominal sepsis. Ann Surg 1982;195:19-24.

13. Smith JA, Bell GA, Murphy J, et al. Evaluation of the use of a protocol in the antimicrobial treatment of intra-abdominal sepsis. J Hosp Infect 1985;6:60-64.

14. Taylor E, Dev V, Shah D, et al. Complicated appendicitis: is there a minimum intravenous antibiotic requirement? A prospective randomized trial. Am Surg 2000;66:887-890.

15. Visser MR, Bosscha K, Olsman J, et al. Predictors of recurrence of fulminant bacterial peritonitis after discontinuation of antibiotics in open management of the abdomen. Eur J Surg 1998;164:825-829.

16. Malangoni MA, Condon RE, Spiegel CA. Treatment of intra-abdominal infections is appropriate with single-agent or combination antibiotic therapy. Surgery 1985;98:648-655.

17. Yellin AE, Heseltine PNR, Berne TV, et al. The role of Pseudomonas species in patients treated with ampicillin and sulbactam for gangrenous and perforated appendicitis. Surg Gynecol Obstet 1985;161:303-307.

18. Study Group of Intraabdominal Infections. A randomized controlled trial of ampicillin plus sulbactam vs. gentamicin plus clindamycin in the treatment of intraabdominal infections. A preliminary report. Rev Infect Dis 1986;8:S583-S588.

19. Lewis RT, Duma RJ, Echols RM, et al. Comparative study of cefotetan and cefoxitin in the treatment of in- tra-abdominal infections. Am J Obstet Gynecol 1988; 158:728-735.

20. Wilson SE, Boswick JA Jr, Duma RJ, et al. Cephalosporin therapy in intraabdominal infections. A multicenter randomized, comparative study of cefotetan, moxalactam, and cefoxitin. Am J Surg 1988; 155:61-66.

21. Holloway WJ, Winslow DL, Reinhardt JF. Cefmetazole treatment of intra-abdominal infection. J Antimicrob Chemother 1989;23(Suppl D):47-54.

22. Anonymous. A randomized multicentre trial of pefloxacin plus metronidazole and gentamicin plus metronidazole in the treatment of severe intra-abdominal infections. Reports from a Swedish Study Group. J Antimicrob Chemother 1990;26(Suppl B):173-180.

23. Bubrick MP, Heim-Duthoy KL, Yellin AE, et al. Ceftazidime/clindamycin versus tobramycin/clindamycin in the treatment of intra-abdominal infections. Am Surg 1990;56:613-617.

24. Jauregui LE, Appelbaum PC, Fabian TC, et al. A randomized clinical study of cefoperazone and sulbactam versus gentamicin and clindamycin in the treatment of intra-abdominal infections. J Antimicrob Chemother 1990;25:423-433.

25. Kooi GH, Pit S. Ceftazidime/metronidazole versus netilmicin/metronidazole in the treatment of perforated appendicitis in children. Clin Ther 1990;12:54-60.

26. Poenaru D, De Santis M, Christou NV. Imipenem versus tobramycin-antianaerobe antibiotic therapy in intra-abdominal infections. Can J Surg 1990;33:415-422.

27. Solomkin JS, Dellinger EP, Christou NV, et al. Results of a multicenter trial comparing imipenem/cilastatin to tobramycin/clindamycin for intra-abdominal infections. Ann Surg 1990;212:581-591.

28. Luke M, Iversen J, Søndergaard J, et al. Ceftriaxone/ metronidazole is more effective than ampicillin/ netilmicin/metronidazole in the treatment of bacterial peritonitis. Eur J Surg 1991;157:397-401.

29. Meller JL, Reyes HM, Loeff DS, et al. One-drug versus two-drug antibiotic therapy in pediatric perforated appendicitis: a prospective randomized study. Surgery 1991;110:764-768.

30. Paakkonen M, Alhava EM, Huttunen $R$, et al. Piperacillin compared with cefuroxime plus metronidazole in diffuse peritonitis. Eur J Surg 1991;157: 535-537.

31. Schropp KP, Kaplan S, Golladay ES, et al. A randomized clinical trial of ampicillin, gentamicin and clindamycin versus cefotaxime and clindamycin in children with ruptured appendicitis. Surg Gynecol Obstet 1991;172:351-356.

32. Sirinek KR, Levine BA. A randomized trial of ticarcillin and clavulanate versus gentamicin and clindamycin in patients with complicated appendicitis. Surg Gynecol Obstet 1991;172(Suppl):30-35.

33. Williams RR, Hotchkin D. Aztreonam plus clindamycin versus tobramycin plus clindamycin in the treatment of intraabdominal infections. Rev Infect Dis 1991;13:S629-S633. 
34. Yoshioka K, Youngs DJ, Keighley MR. A randomized prospective controlled study of ciprofloxacin with metronidazole versus amoxicillin/clavulanic acid with metronidazole in the treatment of intra-abdominal infection. Infection 1991;19:25-29.

35. Brismar B, Malmborg AS, Tunevall G, et al. Piperacillin-tazobactam versus imipenem-cilastatin for treatment of intra-abdominal infections. Antimicrob Agents Chemother 1992;36:2766-2773.

36. Eckhauser FE, Knol JA, Raper SE, et al. Efficacy of two comparative antibiotic regimens in the treatment of serious intra-abdominal infections: results of a multicenter study. Clin Ther 1992;14:97-109.

37. Berne TV, Yellin AE, Appleman MD, et al. A clinical comparison of cefepime and metronidazole versus gentamicin and clindamycin in the antibiotic management of surgically treated advanced appendicitis. Surg Gynecol Obstet 1993;177(Suppl):18-22.

38. de Groot HG, Hustinx PA, Lampe AS, et al. Comparison of imipenem/cilastatin with the combination of aztreonam and clindamycin in the treatment of intra-abdominal infections. J Antimicrob Chemother 1993;32:491-500.

39. Kanellakopoulou K, Giamarellou H, Papadothomakos $\mathrm{P}$, et al. Meropenem versus imipenem/ cilastatin in the treatment of intraabdominal infections requiring surgery. Eur J Clin Microbiol Infect Dis 1993;12:449-453.

40. Niinikoski J, Havia T, Alhava E, et al. Piperacillin/ tazobactam versus imipenem/cilastatin in the treatment of intra-abdominal infections. Surg Gynecol Obstet 1993;176:255-261.

41. Polk HC Jr, Fink MP, Laverdiere M, et al. Prospective randomized study of piperacillin/tazobactam therapy of surgically treated intra-abdominal infection. Am Surg 1993;59:598-605.

42. Walker AP, Nichols RL, Wilson RF, et al. Efficacy of a beta-lactamase inhibitor combination for serious intra-abdominal infections. Ann Surg 1993;217:115-121.

43. Yellin AE, Berne TV, Heseltine PN, et al. Prospective randomized study of two different doses of clindamycin admixed with gentamicin in the management of perforated appendicitis. Am Surg 1993;59: 248-255.

44. Barboza E, del Castillo M, Yi A, et al. Clindamycin plus amikacin versus clindamycin plus aztreonam in established intraabdominal infections. Surgery 1994; 116:28-35.

45. Greenberg RN, Cayavec P, Danko LS, et al. Comparison of cefoperazone plus sulbactam with clindamycin plus gentamicin as treatment for intra-abdominal infections. J Antimicrob Chemother 1994;34: 391-401.

46. Hopkins JA, Wilson SE, Bobey DG. Adjunctive antimicrobial therapy for complicated appendicitis: bacterial overkill by combination therapy. World J Surg 1994;18:933-938.

47. Brismar B, Malmborg AS, Tunevall G, et al. Meropenem versus imipenem/cilastatin in the treatment of intraabdominal infections. J Antimicrob Chemother 1995; 35:139-148.
48. Condon RE, Walker AP, Sirinek KR, et al. Meropenem versus tobramycin plus clindamycin for treatment of intraabdominal infections: results of a prospective, randomized, double-blind clinical trial. Clin Infect Dis 1995;21:544-550.

49. Dougherty SH, Sirinek KR, Schauer PR, et al. Ticarcillin/clavulanate compared with clindamycin/gentamicin (with or without ampicillin) for the treatment of intra-abdominal infections in pediatric and adult patients. Am Surg 1995;61:297-303.

50. Geroulanos SJ, the Meropenem Study Group. Meropenem versus imipenem/cilastatin in intra-abdominal infections requiring surgery. J Antimicrob Chemother 1995;36(Suppl A):191-205.

51. Huizinga WKJ, Warren BL, Baker LW, et al. Antibiotic monotherapy with meropenem in the surgical management of intra-abdominal infections. J Antimicrob Chemother 1995;36(Suppl A):179-189.

52. Shyr YM, Lui WY, Su CH, et al. Piperacillin/tazobactam in comparison with clindamycin plus gentamicin in the treatment of intra-abdominal infections. Chung Hua I Hsueh Tsa Chih-Chinese Med J 1995;56: 102-108.

53. Angerås $\mathrm{MH}$, Darle $\mathrm{N}$, Hamnström $\mathrm{K}$, et al. A comparison of imipenem/ cilastatin with the combination of cefuroxime and metronidazole in the treatment of intra-abdominal infections. Scand J Infect Dis 1996; 28(5):513-518.

54. Berne TV, Yellin AE, Appleman MD, et al. Meropenem versus tobramycin with clindamycin in the antibiotic management of patients with advanced appendicitis. J Am Coll Surg 1996;182:403-407.

55. Christou NV, Turgeon P, Wassef R, et al. Management of intra-abdominal infections. The case for intraoperative cultures and comprehensive broad-spectrum antibiotic coverage. Arch Surg 1996;131:11931201.

56. Solomkin JS, Reinhart HH, Dellinger EP, et al. Results of a randomized trial comparing sequential intravenous/oral treatment with ciprofloxacin plus metronidazole to imipenem/cilastatin for intra-abdominal infections. Ann Surg 1996;223:303-315.

57. Barie PS, Vogel SB, Dellinger EP, et al. A randomized, double-blind clinical trial comparing cefepime plus metronidazole with imipenem-cilastatin in the treatment of complicated intra-abdominal infections. Arch Surg 1997;132:1294-1302.

58. Basoli A, Meli EZ, Mazzocchi P, et al. Imipenem/ cilastatin (1.5 g daily) versus meropenem ( $3.0 \mathrm{~g}$ daily) in patients with intra-abdominal infections: results of a prospective, randomized, multicentre trial. Scand J Infect Dis 1997;29:503-508.

59. Chang DC, Wilson SE. Meta-analysis of the clinical outcome of carbapenem monotherapy in the adjunctive treatment of intra-abdominal infections. Am J Surg 1997;174:284-290.

60. Ciftci AO, Tanyel FC, Büyükpamukçu N, et al. Comparative trial of four antibiotic combinations for perforated appendicitis in children. Eur J Surg 1997;163: 591-596. 
61. Kempf P, Bauernfeind A, Müller A, et al. Meropenem monotherapy versus cefotaxime plus metronidazole combination treatment for serious intra-abdominal infections. Infection 1996;24:473-479.

62. Wilson SE. Results of randomized, multicenter trial of meropenem versus clindamycin/tobramycin for the treatment of intra-abdominal infections. Clin Infect Dis 1997;24(Suppl 2):S197-206.

63. Collins MD, Dajani AS, Kim KS, et al. Comparison of ampicillin/sulbactam plus aminoglycoside vs. ampicillin plus clindamycin plus aminoglycoside in the treatment of intraabdominal infections in children. Pediatr Infect Dis J 1998;17(3 Suppl):S15-S18.

64. Donahue PE, Smith DL, Yellin AE, et al. Trovafloxacin in the treatment of intra-abdominal infections: results of a double-blind, multicenter comparison with imipenem/ cilastatin. Am J Surg 1998;176(Suppl 6A): 53S-61S.

65. Jaccard C, Troillet N, Harbarth S, et al. Prospective randomized comparison of imipenem-cilastatin and piperacillin-tazobactam in nosocomial pneumonia or peritonitis. Antimicrob Agents Chemother 1998;42: 2966-2972.

66. Allo MD, Bennion RS, Kathir K, et al. Ticarcillin/ clavulanate versus imipenem/cilistatin for the treatment of infections associated with gangrenous and perforated appendicitis. Am Surg 1999;65:99-104.

67. Ohlin B, Cederberg A, Forssell H, et al. Piperacillin/ tazobactam compared with cefuroxime/metronidazole in the treatment of intra-abdominal infections. Eur J Surg 1999;165:875-884.

68. Zanetti G, Harbarth SJ, Trampuz A, et al. Meropenem ( $1.5 \mathrm{~g} /$ day) is as effective as imipenem/ cilastatin (2 $\mathrm{g} /$ day) for the treatment of moderately severe intraabdominal infections. Int J Antimicrob Agents 1999; 11:107-113.

69. Cohn SM, Lipsett PA, Buchman TG, et al. Comparison of intravenous/oral ciprofloxacin plus metronidazole versus piperacillin/tazobactam in the treatment of complicated intraabdominal infections. Ann Surg 2000;232:254-256.

70. Röhrborn A, Wacha H, Schöffel U, et al. Coverage of enterococci in community-acquired secondary peritonitis: results of a randomized trial. Surg Infect 2000;1:95-107.

71. Torres AJ, Valladares LD, Jover JM, et al. Cefminox versus metronidazole plus gentamicin in intra-abdominal infections: a prospective randomized controlled clinical trial. Infection 2000;28:318-322.

72. Solomkin JS, Yellin E, Rotstein OD, et al. Results of a double-blind, randomized comparative phase III trial of ertapenem vs. piperacillin/tazobactam in the treatment of complicated intraabdominal infections. Ann Surg (in press).

73. Hatala R, Dinh T, Cook DJ. Once-daily aminoglycoside dosing in immunocompetent adults: a metaanalysis. Ann Intern Med 1996;124:717-725.

74. Hollender LF, Bahnini J, De Manzini N, et al. A multicentric study of netilmicin once daily versus thrice daily in patients with appendicitis and other intra-ab- dominal infections. J Antimicrob Chemother 1989;23: 773-783.

75. de Vries PJ, Verkooyen RP, Leguit P, et al. Prospective randomized study of once-daily versus thricedaily netilmicin regimens in patients with intraabdominal infections. Eur J Clin Microbiol Infect Dis 1990;9:161-168.

76. Mosdell DM, Morris DM, Voltura A, et al. Antibiotic treatment for surgical peritonitis. Ann Surg 1991;214: 543-549.

77. Dellinger EP, Wertz MJ, Meakins JL, et al. Surgical infection stratification system for intra-abdominal infection. Arch Surg 1985;120:21-29.

78. Christou NV, Barie PS, Dellinger EP, et al. Surgical Infection Society intra-abdominal infection study. Prospective evaluation of management techniques and outcome. Arch Surg 1993;128:193-199.

79. Bohnen JM, Mustard RA, Schouten BD. Steroids, APACHE II score, and the outcome of abdominal infection. Arch Surg 1994;129:33-37.

80. Schoeffel U, Jacobs E, Ruf G, et al. Intraperitoneal micro-organisms and the severity of peritonitis. Eur J Surg 1995;161:501-508.

81. Pacelli F, Doglietto GB, Alfieri S, et al. Prognosis in intra-abdominal infections. Multivariate analysis on 604 patients. Arch Surg 1996;131:641-645.

82. Ohmann C, Hau T. Prognostic indices in peritonitis. Hepatogastroenterology 1997;44:937-946.

83. Wacha H, Hau T, Dittmer R, et al. Risk factors associated with intraabdominal infections: a prospective multicenter study. Langenbecks Arch Surg 1999;384: 24-32.

84. Hopkins JA, Lee JCH, Wilson SE. Susceptibility of intra-abdominal isolates at operation: a predictor of postoperative infection. Am Surg 1993;59:791-796.

85. Montravers P, Gauzit R, Muller C, et al. Emergence of antibiotic resistant bacteria in cases of peritonitis after intraabdominal surgery affects the efficacy of empirical antimicrobial therapy. Clin Infect Dis 1996; 23:486-494.

86. Cometta A, Baumgartner JD, Lew D, et al. Prospective randomized comparison of imipenem monotherapy with imipenem plus netilmicin for treatment of severe infections in nonneutropenic patients. Antimicrob Agents Chemother 1994;38:1309-1313.

87. Hoogkamp-Korstanje JA. Ciprofloxacin vs. cefotaxime regimens for the treatment of intra-abdominal infections. Infection 1995;23:278-282.

88. Dupont H, Carbon C, Carlet J, The Severe Generalized Peritonitis Study Group. Monotherapy with a broad-spectrum beta-lactam is as effective as its combination with an aminoglycoside in treatment of severe generalized peritonitis: a multicenter randomized controlled trial. Antimicrob Agents Chemother 2000;44:2028-2033.

89. Burnett RJ, Haverstock DC, Dellinger EP, et al. Definition of the role of enterococcus in intraabdominal infection: analysis of a prospective randomized trial. Surgery 1995;118:716-723.

90. Solomkin JS, Flohr AB, Quie PG, et al. The role of Can- 
dida in intraperitoneal infections. Surgery 1980;88: 524-530.

91. Calandra T, Bille J, Schneider R, et al. Clinical significance of Candida isolated from peritoneum in surgical patients. Lancet 1989;2(8677):1437-1440.

92. Eggiman P, Francioli P, Bille J, et al. Fluconazole prophylaxis prevents intra-abdominal candidiasis in high-risk surgical patients. Crit Care Med 1999;27: 1066-1072.

93. Abele-Horne M, Kopp A, Sternberg U, et al. A randomized study comparing fluconazole with amphotericin B/5-flucytosine for the treatment of systemic Candida infections in intensive care patients. Infection 1996;24:426-432.

94. Rotstein OD, Pruett TL, Simmons RL. Microbiologic features and treatment of persistent peritonitis in patients in the intensive care unit. Can J Surg 1986;29: 247-250.
95. Nathens AB, Rotstein OD, Marshall JC. Tertiary peritonitis: clinical features of a complex nosocomial infection. World J Surg 1998;22:158-163.

96. Solomkin JS. Antibiotic resistance in postoperative infections. Crit Care Med 2001;29(Suppl):N97-N99.

Address reprint requests to: John E. Mazuski, M.D., Ph.D. Department of Surgery, Campus Box 8109 Washington University School of Medicine 660 S. Euclid Ave. St. Louis, MO 63110-1093

E-mail: mazuskij@msnotes.wustl.edu 